\title{
Nonextensive statistical effects in protoneutron stars
}

\author{
A. Lavagno, D. Pigato \\ Dipartimento di Fisica, Politecnico di Torino, I-10129 Torino, Italy and \\ Istituto Nazionale di Fisica Nucleare (INFN), Sezione di Torino, I-10126 Torino, Italy \\ Received: date / Revised version: date
}

\begin{abstract}
We investigate the bulk properties of protoneutron stars in the framework of a relativistic mean field theory based on nonextensive statistical mechanics, characterized by power-law quantum distributions. We study the relevance of nonextensive statistical effects on the $\beta$-stable equation of state at fixed entropy per baryon, in presence and in absence of trapped neutrinos, for nucleonic and hyperonic matter. We show that nonextensive statistical effects could play a crucial role in the structure and in the evolution of the protoneutron stars also for small deviations from the standard Boltzmann-Gibbs statistics.
\end{abstract}

PACS. 21.65.-f - 26.60.-c - 97.60.-s - 05.90.+m

\section{Introduction}

A protoneutron star (PNS) is born immediately after the gravitational collapse of a massive star $\left(M \approx 10 \div 20 M_{\odot}\right)$. During the first seconds of its evolution a PNS is a very hot, lepton rich and beta-stable object, with a temperature of a few tens of $\mathrm{MeV}$ and a lepton concentration typical of the pre-supernova matter [1]. Nearly all of its binding energy is in the form of neutrinos trapped inside the stellar structure. In particular, neutrinos at the star core are basically of the $\nu_{e}$ type and have a typically energy of about $E_{\nu} \approx 200 \div 300 \mathrm{MeV} 2$. Shortly after the PNS formation, during the cooling, neutrinos escape through the star structure and bring out in few seconds nearly all of its binding energy. The total luminosity of this process depends from several factors, the most important are the total mass of the compact object and the neutrino opacity. During this process, called Kelvin-Helmholtz epoch, the PNS evolves in a quasi hydrostatic equilibrium, from a lepton rich and hot object to a cold neutrino-free compact star [3]. From a measure of neutrinos luminosity and average energy, which are the most important astrophysical observable in the study of the stellar structure, it is in principle possible to deduce the total binding energy and, therefore, the total baryonic mass of the star [4. However, neutrinos are not only important probes in the study of newborn PNS, but have also relevant consequences on the chemical composition and in the maximum mass of the compact object. The presence of neutrinos in fact alters significantly the chemical composition of the star, varying the formation threshold of different particle species (in general the appearance of non-leptonic negative particles is delayed when neutrinos are present). The changes in the maximum mass, due to neutrinos trapping, are normally greater than those due to finite temperature effects $[2,3$ 4, 5.
From a more theoretical point of view, it appears evident that the knowledge of the nuclear EOS plays a crucial role in the determination of the structure and in the evolution of the PNS 6]. The processes related to strong interaction should in principle be described by quantum chromodynamics. However, in the energy density range reached in the compact stars, strongly non-perturbative effects in the complex theory of QCD are not negligible. The central core of a compact star does not at all resemble a quasi-ideal gas of hadrons because strongly dynamical correlations are present, including long-range interactions [7,8. In the absence of a converging method to approach QCD at finite density one often turns to (effective and phenomenological) model investigations.

Recently, there is an increasing evidence that the nonextensive statistical mechanics, characterized by power-law quantum equilibrium distributions, can be considered as an appropriate basis to deal with physical phenomena where strong dynamical correlations, long-range interactions, anomalous diffusion and microscopic memory effects take place $9,10,11,12$. A considerable variety of physical applications involve a quantitative agreement between experimental data and theoretical models based on the generalized thermostatistics. In particular, in the last years several authors have outlined the relevance of nonextensive statistical mechanics effects in high energy physics and astrophysical problems [13,14, 15, 16, 17, 18, 19, 20, 21, $22,23,24,25]$. The existence of nonextensive statistical effects should strongly affect the finite temperature and density nuclear EOS [26, 27]. In fact, by varying temperature and density, the EOS reflects in terms of the macroscopic thermodynamical variables the microscopic interactions in different nuclear matter regimes.

From a phenomenological point of view, in this work we plan to investigate the relevance of nonextensive sta- 
tistical effects on the main physical properties of PNS and their related astrophysical implications. At this scope, we are going to explore different stages of the PNS evolution. The first stage corresponds to an entropy per baryon equal to one, in which neutrinos are trapped and strongly influence the chemical composition of the PNS. After a short time, of about $10 \div 15 \mathrm{~s}$, the temperature of the PNS fast rises up until it reaches a value of $T \simeq 45 \div 80 \mathrm{MeV}$, it depends again on the chemical composition 28. This stage is called deleptonization era and corresponds to the maximum heating and entropy per baryon $(s=2)$. This is the phase, at high temperature and high baryon density, in which the presence of nonextensive effects may alter more sensibly the thermodynamical and mechanical proprieties of the PNS. Furthermore, let us observe that we are going to consider only hadronic composition of the PNS, and we do not take in consideration possible formation of a mixed hadron-quark phase region or a quark core inside it. This choice is principally due to the fact that the presence of trapped neutrinos delays the on-set of strange particles and also possible formation of quark matter [2, 3. As a consequence, the appearance of a mixed phase or a quark core could be shifted at the end, or nearly at the end of the deleptonization era 29 .

The paper is organized as follows. In Section 2, we briefly introduce the basic formalism of the nonextensive statistics. In Section [3, we study the nonextensive hadronic EOS in the context of a relativistic mean field model, in presence and in absence of neutrinos and hyperons. In Section 4 , we show the main results in the particles concentrations and bulk stellar properties in the presence of nonextensive statistical effects. Finally, we summarize our conclusions in Section 5 .

\section{Basic assumptions in nonextensive statistics}

Starting point of the nonextensive statistical mechanics, introduced by Tsallis, is the following definition of a $q$ deformed entropy functional [9, 10, 11]:

$$
S_{q}[f]=\frac{1}{q-1}\left(1-\int[f(\mathbf{x})]^{q} d \Omega\right),\left(\int f(\mathbf{x}) d \Omega=1\right)(1)
$$

where $f(\mathbf{x})$ stands for a normalized probability distribution, $\mathbf{x}$ and $d \Omega$ denoting, respectively, a generic point and the volume element in the corresponding phase space (here and in the following we set the Boltzmann and the Planck constant equal to unity). The generalized entropy has the usual properties of positivity, equiprobability, concavity and irreversibility, preserves the whole mathematical structure of thermodynamics (Legendre transformations). The real parameter $q$ determines the degree of nonadditivity exhibited by the entropy form (1) and in the limit $q \rightarrow 1$, becomes additive and reduces to the standard Boltzmann-Gibbs entropy.

A peculiarity of the Tsallis generalized thermostatistics is that if we have two statistically independent subsystems $A$ e $B$, described, respectively, by individual probability density $f^{(A)}$ and $f^{(B)}$ and we call $f^{(A+B)}\left(\mathbf{x}_{A}, \mathbf{x}_{B}\right)=$
$f^{(A)}\left(\mathbf{x}_{A}\right) f^{(B)}\left(\mathbf{x}_{B}\right)$ the joint probability density of a composite system $A+B$, the nonadditive (nonextensive) character of $S_{q}$ is summarized in the relation [1]

$$
\begin{aligned}
S_{q}\left[f^{(A+B)}\right]= & \left.S_{q}\left[f^{(A)}\right]+S_{q}\left[f^{(B)}\right]\right) \\
& +(1-q) S_{q}\left[f^{(A)}\right] S_{q}\left[f^{(B)}\right] .
\end{aligned}
$$

In the limit $q \rightarrow 1$, the above equation reduces to the wellknown additivity (extensivity) relation of the BoltzmannGibbs logarithmic entropy. Here, the word nonextensive should be associated with the fact that the total energy of long-range-interacting mechanical systems is nonextensive, in contrast with the case of short-range-interacting systems, whose total energy is extensive in the thermodynamical sense [11.

Second crucial assumption on nonextensive statistics is the introduction of the $q$-mean value (or escort mean value) of a physical observable $A(\mathbf{x})[9,10,11$ :

$$
\langle A\rangle_{q}=\frac{\int A(\mathbf{x})[f(\mathbf{x})]^{q} d \Omega}{\int[f(\mathbf{x})]^{q} d \Omega} .
$$

The probability distribution can be obtained maximizing the measure $S_{q}$ under appropriate constraints related to the previous $q$-mean value definition. In this context, it is important to observe that the Tsallis classical distribution can be seen as a superposition of Boltzmann distributions with different temperatures which have a mean value corresponding to the temperature appearing in the Tsallis distribution. The nonextensive $q$ parameter is related to the fluctuation in the temperature and describes the spread around the average value of the Boltzmann temperature [16].

Following the above prescriptions, it is possible to obtain the associate quantum mean occupation number of particles species $i$ in a grand canonical ensemble. For a dilute gas of particles and for small deviations from the standard statistics $(q \approx 1)$, it can be written as 30,31 .

$$
n_{i}=\frac{1}{\tilde{e}_{q}\left(\beta\left(E_{i}-\mu_{i}\right)\right) \pm 1},
$$

where $\beta=1 / T$ and the sign $( \pm 1)$ is for fermions and bosons respectively. Furthermore, in Eq.(44), following Ref. [31, for $q>1$, we have

$$
\tilde{e}_{q}(x)= \begin{cases}{[1+(q-1) x]^{1 /(q-1)}} & \text { if } x>0 \\ {[1+(1-q) x]^{1 /(1-q)}} & \text { if } x \leq 0\end{cases}
$$

whereas, for $q<1$,

$$
\tilde{e}_{q}(x)= \begin{cases}{[1+(q-1) x]^{1 /(q-1)}} & \text { if } x \leq 0 \\ {[1+(1-q) x]^{1 /(1-q)}} & \text { if } x>0\end{cases}
$$

Naturally, for $q \rightarrow 1$ the above quantum distribution reduces to the standard Fermi-Dirac and Bose-Einstein distribution. Let us observe nonextensive statistical effects vanishes approaching to zero temperature. On the other hand, the nonextensive statistics entails a sensible difference on the power-law particle distribution shape in the high energy region with respect to the standard statistics. Hence, nonextensive effects could play an important role in the finite temperature and high baryon density PNS evolution. 


\section{Nonextensive hadronic equation of state}

In this Section, we study the nonextensive hadronic EOS in the framework of a relativistic mean field theory in which baryons interact through the nuclear force mediated by the exchange of virtual isoscalar-scalar $(\sigma)$, isoscalar-vector $(\omega)$ and isovector-vector $(\rho)$ mesons fields 32 , 33, 34. In our analysis we include all the baryon octet in order to reproduce the chemical composition of the PNS at high baryon chemical potential. We also take into account of leptons particle by fixing the lepton fraction $Y_{L}=Y_{e}+Y_{\nu_{e}}=\left(\rho_{e}+\rho_{\nu_{e}}\right) / \rho_{B}$, where $\rho_{e}, \rho_{\nu_{e}}$ and $\rho_{B}$ are the electron, neutrino and baryon number densities, respectively. This is because, in the first stage of PNS evolution, electrons and neutrinos are trapped inside the stellar matter and, therefore, the lepton number must be conserved until neutrinos escape out of the PNS [2,4,5].

The Lagrangian density can be written in term of the hadronic [34,35] plus leptonic component, as follow:

$$
\begin{aligned}
& \mathcal{L}_{\text {tot }}=\mathcal{L}_{H}+\mathcal{L}_{l}=\sum_{B} \bar{\psi}_{B}\left[i \gamma_{\mu} \partial^{\mu}-\left(M_{B}-g_{\sigma B} \sigma\right)\right. \\
& \left.-g_{\omega B} \gamma_{\mu} \omega^{\mu}-g_{\rho B} \gamma^{\mu} \boldsymbol{\tau} \cdot \boldsymbol{\rho}_{\mu}\right] \psi_{B}+\frac{1}{2}\left(\partial_{\mu} \sigma \partial^{\mu} \sigma-m_{\sigma}^{2} \sigma^{2}\right) \\
& -U(\sigma)+\frac{1}{2} m_{\omega}^{2} \omega_{\mu} \omega^{\mu}+\frac{1}{2} m_{\rho}^{2} \boldsymbol{\rho}_{\mu} \cdot \boldsymbol{\rho}^{\mu}-\frac{1}{4} F_{\mu \nu} F^{\mu \nu} \\
& -\frac{1}{4} \boldsymbol{G}_{\mu \nu} \boldsymbol{G}^{\mu \nu}+\sum_{l} \bar{\psi}_{l}\left[i \gamma_{\mu} \partial^{\mu}-m_{l}\right] \psi_{l}
\end{aligned}
$$

where the sums over $B$ and $l$ are over the baryon octet and lepton particles, respectively. The field strength tensors for the vector mesons are given by the usual expressions $F_{\mu \nu} \equiv \partial_{\mu} \omega_{\nu}-\partial_{\nu} \omega_{\mu}, \boldsymbol{G}_{\mu \nu} \equiv \partial_{\mu} \boldsymbol{\rho}_{\nu}-\partial_{\nu} \boldsymbol{\rho}_{\mu}$, and $U(\sigma)$ is a nonlinear potential of $\sigma$ meson

$$
U(\sigma)=\frac{1}{3} a \sigma^{3}+\frac{1}{4} b \sigma^{4},
$$

usually introduced to achieve a reasonable compression modulus for equilibrium nuclear matter.

The field equations in a mean field approximation are

$$
\begin{aligned}
& \left(i \gamma_{\mu} \partial^{\mu}-\left(M-g_{\sigma B} \sigma\right)-g_{\omega B} \gamma^{0} \omega-g_{\rho B} \gamma^{0} \tau_{3} \rho\right) \psi=0 \\
& m_{\sigma}^{2} \sigma+a \sigma^{2}+b \sigma^{3}=g_{\sigma B}<\bar{\psi} \psi>=g_{\sigma B} \rho_{S} \\
& m_{\omega}^{2} \omega=g_{\omega B}<\bar{\psi} \gamma^{0} \psi>=g_{\omega B} \rho_{B} \\
& m_{\rho}^{2} \rho=g_{\rho B}<\bar{\psi} \gamma^{0} \tau_{3} \psi>=g_{\rho B} \rho_{I}
\end{aligned}
$$

where $\sigma=\langle\sigma\rangle, \omega=\left\langle\omega^{0}\right\rangle$ and $\rho=\left\langle\rho_{3}^{0}\right\rangle$ are the nonvanishing expectation values of mesons fields, $\rho_{I}$ is the total isospin density, $\rho_{B}$ and $\rho_{S}$ are the baryon density and the baryon scalar density, respectively. They are given by

$$
\begin{aligned}
& \rho_{B}=2 \sum_{i=B} \int \frac{\mathrm{d}^{3} k}{(2 \pi)^{3}}\left[n_{i}(k)-\bar{n}_{i}(k)\right], \\
& \rho_{S}=2 \sum_{i=B} \int \frac{\mathrm{d}^{3} k}{(2 \pi)^{3}} \frac{M_{i}^{*}}{E_{i}^{*}}\left[n_{i}^{q}(k)+\bar{n}_{i}^{q}(k)\right],
\end{aligned}
$$

where $n_{i}(k)$ and $\bar{n}_{i}(k)$ are the $q$-deformed fermion particle and antiparticle distributions given in Eq.s(4)-(6); for example, for $q>1$ and $\beta\left(E_{i}^{*}-\left|\mu_{i}^{*}\right|\right)>0$, we have

$$
\begin{aligned}
& n_{i}(k)=\frac{1}{\left[1+(q-1) \beta\left(E_{i}^{*}(k)-\mu_{i}^{*}\right)\right]^{1 /(q-1)}+1}, \\
& \bar{n}_{i}(k)=\frac{1}{\left[1+(q-1) \beta\left(E_{i}^{*}(k)+\mu_{i}^{*}\right)\right]^{1 /(q-1)}+1} .
\end{aligned}
$$

The nucleon effective energy is defined as $E_{i}{ }^{*}(k)=$ $\sqrt{k^{2}+M_{i}^{* 2}}$, where $M_{i}^{*}=M_{i}-g_{\sigma B} \sigma$. The effective chemical potentials $\mu_{i}^{*}$ are given in terms of the meson fields as follows

$$
\mu_{i}^{*}=\mu_{i}-g_{\omega B} \omega-\tau_{3 i B} g_{\rho B} \rho,
$$

where $\mu_{i}$ are the thermodynamical chemical potentials $\left(\mu_{i}=\partial \epsilon / \partial \rho_{i}\right)$.

The further conditions, required for the $\beta$-stable chemical equilibrium and charge neutrality, can be written as

$$
\begin{aligned}
& \mu_{\Lambda}=\mu_{\Sigma^{0}}=\mu_{\Xi^{0}}=\mu_{n}, \\
& \mu_{\Sigma^{-}}=\mu_{\Xi^{-}}=\mu_{n}+\mu_{e}, \\
& \mu_{p}=\mu_{\Sigma^{+}}=\mu_{n}-\mu_{e} \\
& \rho_{p}+\rho_{\Sigma^{+}}-\rho_{\Sigma^{-}}-\rho_{\Xi^{-}}-\rho_{e}=0 .
\end{aligned}
$$

In the case of trapped neutrinos, the new equalities are obtained by the replacement of $\mu_{e} \rightarrow \mu_{e}-\mu_{\nu_{e}}$. The total entropy per baryon is calculated using $s=\left(S_{B}+S_{l}\right) /\left(T \rho_{B}\right)$, where $S_{B}=P_{B}+\epsilon_{B}-\sum_{i=B} \mu_{i} \rho_{i}$ and $S_{l}=P_{l}+\epsilon_{l}-$ $\sum_{i=l} \mu_{i} \rho_{i}$, and the sums are extended over all the baryons and leptons species.

The thermodynamical quantities can be obtained from the thermodynamic potential in the standard way. More explicitly, the baryon pressure $P_{B}$ and the energy density $\epsilon_{B}$ can be written as

$$
\begin{aligned}
P_{B}= & \frac{2}{3} \sum_{i} \int \frac{\mathrm{d}^{3} k}{(2 \pi)^{3}} \frac{k^{2}}{E_{i}^{*}(k)}\left[n_{i}^{q}(k)+\bar{n}_{i}^{q}(k)\right]-\frac{1}{2} m_{\sigma}^{2} \sigma^{2} \\
& -U(\sigma)+\frac{1}{2} m_{\omega}^{2} \omega^{2}+\frac{1}{2} m_{\rho}^{2} \rho^{2} \\
\epsilon_{B}= & 2 \sum_{i} \int \frac{\mathrm{d}^{3} k}{(2 \pi)^{3}} E_{i}^{*}(k)\left[n_{i}^{q}(k)+\bar{n}_{i}^{q}(k)\right]+\frac{1}{2} m_{\sigma}^{2} \sigma^{2} \\
& +U(\sigma)+\frac{1}{2} m_{\omega}^{2} \omega^{2}+\frac{1}{2} m_{\rho}^{2} \rho^{2}
\end{aligned}
$$

The numerical evaluation of the above thermodynamical quantities can be performed if the meson-nucleon and meson-hyperon coupling constants are known. Concerning the meson-nucleon coupling constants $\left(g_{\sigma N}, g_{\omega N}, g_{\rho N}\right)$, they are determined to reproduce properties of equilibrium nuclear matter such as the saturation densities, the binding energy, the symmetric energy coefficient, the compression modulus, and the effective Dirac mass at saturation. Because of a valuable range of uncertainty in the empirical values that must be fitted, especially for the compression modulus and for the effective Dirac mass, in the literature there are different sets of coupling constants. Here and in the following, we focus our investigation by considering the so-called GM3 parameter set (even 
if comparable results can be obtained in other parameter sets) 34,35]. The implementation of hyperon degrees of freedom comes from determination of the corresponding meson-hyperon coupling constants that have been fitted to hypernuclear properties and their specific values are taken from Ref. 36] for the GM3 parameter set.

\section{Results and discussion}

As briefly mentioned in the Introduction, our analysis focus on the relevance of possible nonextensive statistical effects during the first PNS evolution phases. We can ideally divide the evolution into three phases. The first, at the beginning, in which neutrinos are trapped and the entropy per baryon is assumed fixed to $s=1$ and $Y_{L}=0.4$. A second phase, after about $10 \div 15 \mathrm{sec}$, which corresponds to the maximum heating of the star and neutrinos are free $\left(s=2, Y_{\nu_{e}}=0\right)$. Finally, a third phase of cold-catalyzed PNS $\left(s=0, Y_{\nu_{e}}=0\right)$ 28, 37]. Regarding the relevance of nonextensive statistical effects, the most important phase corresponds to the maximum heating, in which the presence of nonextensive statistical effects may play a crucial role in the determination of the PNS chemical composition and related thermodynamical proprieties. In this work we limit ourselves to consider only a small variations from the standard statistics (from $q=0.97$ to $q=1.03$ ).

In Fig. 1, we show the temperature as a function of the baryon density (in units of the saturation nuclear density $\rho_{0}=0.153 \mathrm{fm}^{-3}$ ) and for different values of the nonextensive parameter, in absence $(n p)$ and in presence $(n p H)$ of hyperons. We limit our analysis in the first two phases: in the upper panel, the first leptonic rich state $\left(s=1, Y_{L}=0.4\right)$ and, in the lower panel, the maximum heating phase $\left(s=2, Y_{\nu_{e}}=0\right)$. Indeed in the coldcatalyzed phase $\left(s=0, Y_{\nu_{e}}=0\right)$, the temperature is very low (fews $\mathrm{MeV}$ ), and nonextensive statistical effects may be neglected. In both previous cases, we observe a reduction in temperature in presence of a sub-extensive statistics $(q<1)$ and a general increase for $q>1$. This effect is more remarkable when hyperons are present and for higher values of entropy for baryon. For example, in the maximum heating phase, in presence of hyperons and for $q=1$, the temperature is about $T \cong 37 \mathrm{MeV}$ at baryon density $\rho_{B}=5 \rho_{0}$, whereas, for $q=1.03$ and $q=0.97$, it is approximately equal to $T \cong 51 \mathrm{MeV}$ and $T \cong 21 \mathrm{MeV}$, respectively. Note also that, when hyperons are present, for $s=1$ and $Y_{L}=0.4$, the system evolves in a quasi isothermal configuration above $\rho_{B}=2.5 \div 3 \rho_{0}$. The different behavior in the stellar temperature have important consequences in the PNS evolution and in its particles concentration. Finite temperature properties of matter at high density influence the diffusion of neutrinos, being the neutrino mean free paths strongly temperature dependent 44,37. In particular, neutrino opacity is very sensitive to the inner temperature (in general proportional to $T^{2}$ ) and, therefore, this would affect sensibly the cooling of the PNS, making it longer when $q>1$, and shorter when $q<1$. This matter of fact could have important consequences on the neutrino luminosity, because its drop is
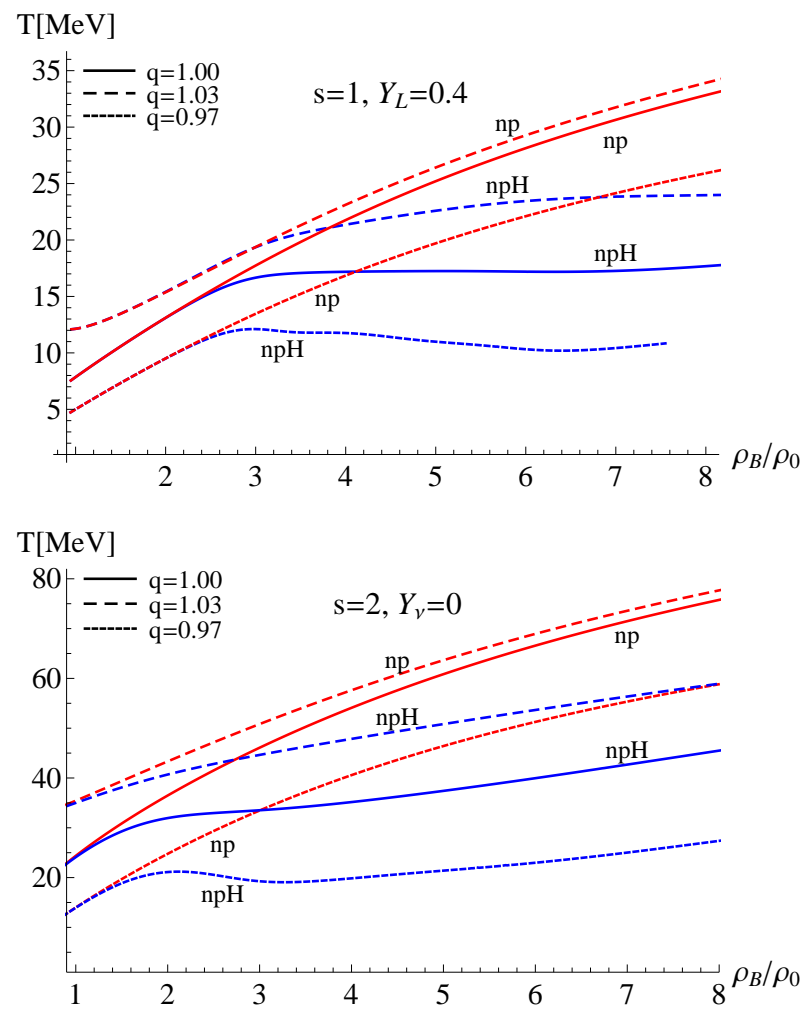

Fig. 1. Temperature as a function of the baryon density (in units of the saturation nuclear density $\rho_{0}$ ) for different values of $q$, entropy per baryon and neutrino fraction (upper panel: $s=1, Y_{L}=0.4$ and lower panel: $s=2, Y_{\nu_{e}}=0$ ). The labels $n p$ and $n p H$ stand for nucleons and nucleons plus hyperons.

associated with the end of this cooling process 3. Consequently, an alteration in the Kelvin-Helmholtz epoch, that does not correspond to the predict direct or modified URCA process, could be an indication of nonextensive statistical effects.

In Fig. 2, is reported the dependence of the pressure from the baryon density, for different values of $q$, in the initial phase: $s=1, Y_{L}=0.4$ (upper panel) and in the maximum heating phase: $s=2$ and $Y_{\nu_{e}}=0$ (lower panel). With the appearance of hyperons, around $\rho_{B}=3 \rho_{0}$, we have a general softening of the EOS. However, due to the low temperature achieved in this phase (see Fig. 1), nonextensive statistical effects do not change significantly the total pressure of the PNS. The situation is somewhat different when we analyze the maximum heating phase. In such a condition, the temperature is higher and nonextensive statistical effects are more relevant, especially when hyperons are present.

As it is well known, the softening of the EOS, due to the appearance of additional fermionic degrees of freedom in the form of hyperons, leads to higher central densities. This matter of fact is, however, influenced from the presence of nonextensive statistical effects. To better focalized this aspect, in Fig. 3 is plotted the central baryon density $\rho_{c}$ in the PNS core corresponding to a total baryon 

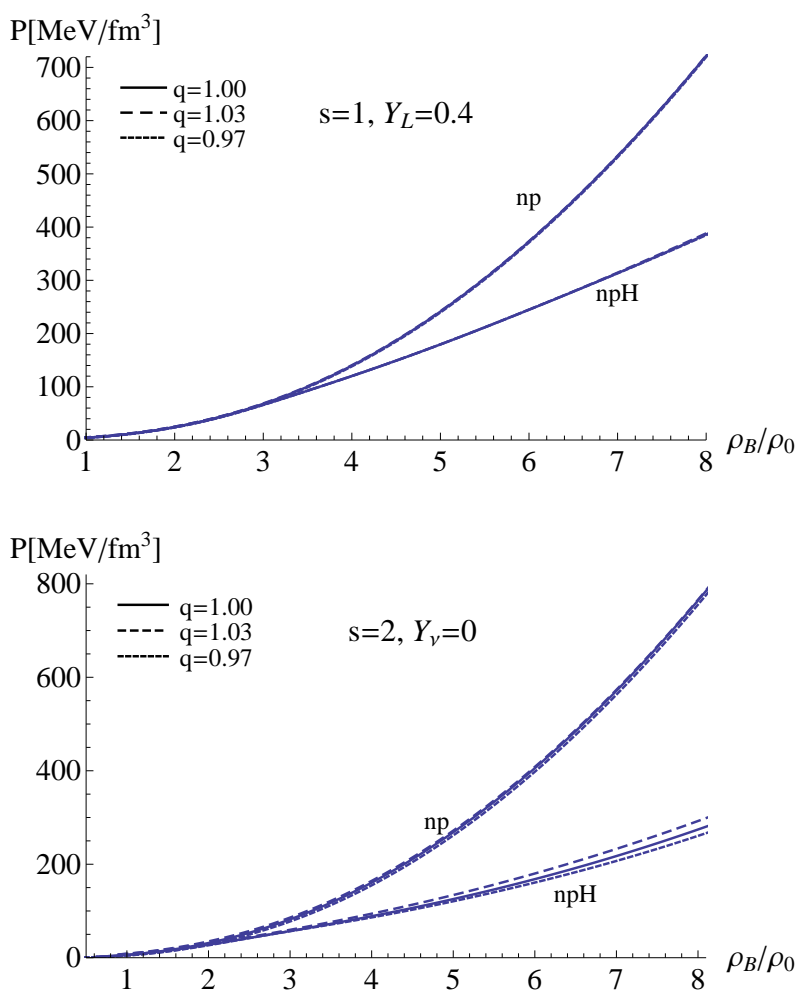

Fig. 2. Total pressure as a function of baryon density (in units of the saturation nuclear density) for different values of $q$ in the lepton rich case: $s=1$ and $Y_{L}=0.4$ (upper panel) and in the maximum heating phase: $s=2$ and $Y_{\nu_{e}}=0$ (lower panel). The labels $n p$ and $n p H$ stand for nucleons and nucleons plus hyperons matter, respectively.

mass $M_{B}$ (in units of the solar mass $M_{\odot}$ ). The case $q>1$ $(q<1)$ implies a general reduction (increase) of the central density at fixed total baryon mass. This effect is emphasized in the maximum heating condition (lower panel). In particular, for $q>1$ and until $M_{B} \approx 1.7 M_{\odot}$, the central densities of hyperons stars $(n p H)$ are lower than the ones corresponding to the standard $(q=1)$ nucleons-only stars $(n p)$. This feature, together the variation of the temperature, can be very relevant in the determination of the specific heat of the stellar matter and, as a consequence, on the neutrino diffusion 4 .

In addition to the above considerations, we investigate the lepton chemical potentials in different PNS conditions because they strongly influence the deleptonization process 4,5. At this scope, in Fig. 4, we show the lepton chemical potentials in lepton rich matter $(s=1$ and $\left.Y_{L}=0.4\right)$ in absence $(n p)$ and in presence $(n p H)$ of hyperon degrees of freedom (higher curves for the electron chemical potential $\mu_{e}$ and lower curves for the neutrino chemical potential $\left.\mu_{\nu_{e}}\right)$. For clarify, we report only the case $q>1$ compared to the standard $(q=1)$ results. It is interesting to observe that for a nucleons-only EOS, super-extensive statistical effects imply a general reduction of the electron and neutrino chemical potentials
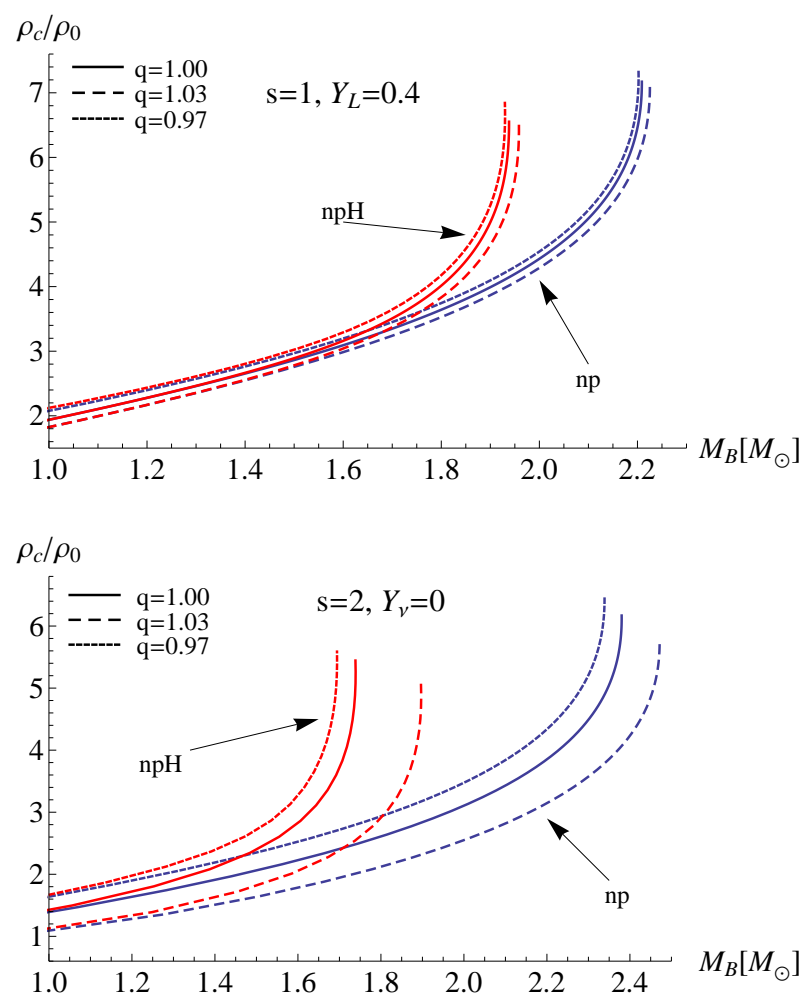

Fig. 3. Central baryon density $\rho_{c}$ (in units of the saturation nuclear density) corresponding to a total stellar baryon mass $M_{B}$ in the lepton rich phase (upper panel) and in the maximum heating phase (lower panel). The labels $n p$ and $n p H$ stand for nucleons and nucleons plus hyperons matter, respectively.

respect to the standard case. Otherwise, in presence of hyperons, a sensible reduction of the electron chemical potential is not accompanied by a reduction of the neutrino chemical potential which retains very similar values to the standard case at high baryon density. Such a feature could have important consequence on the diffusion of the electron neutrinos inside the PNS.

Moreover, the neutrino mean free paths and the matter specific heat depend sensitively on the composition; under degenerate conditions even modest changes to the composition significantly alter the neutrino scattering and absorbtion mean free paths. It is, therefore, relevant to investigate how nonextensive statistical mechanics influences particle compositions in different PNS epochs. In Fig. 5, we report the particle concentrations for $s=1$ and $Y_{L}=0.4$ in absence (upper panel) and in presence (lower panel) of hyperons for different values of $q$. It is well known that the presence of trapped neutrinos significantly alter the protons and the electrons abundance and strongly influence the threshold of hyperons formation 2 , 4.5. In absence of hyperons, nonextensive statistical effects do not play a significantly role, in fact the particle concentrations are almost the same. The situation changes when we include hyperon degrees of freedom. The presence of sub-extensive effects $(q<1)$ slightly lowers the neutri- 


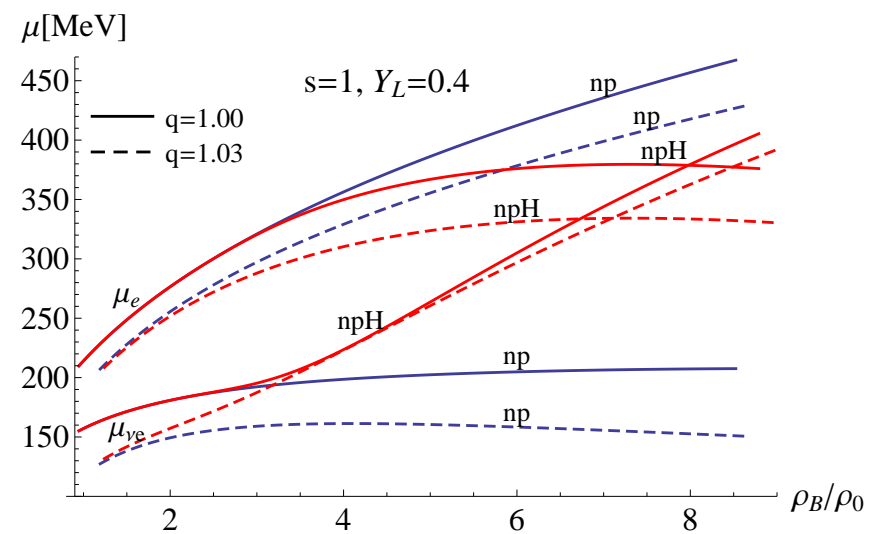

Fig. 4. Lepton chemical potentials in lepton rich matter as a function of the baryon density (in units of the nuclear saturation density). The labels $n p$ and $n p H$ stand for nucleons and nucleons plus hyperons matter, respectively.

nos concentration, while increases the neutrons and the electrons ones. Moreover, hyperons start later and their concentration are in general decreased, excepted for the $\Lambda$ particles ratio, which becomes greater after $\rho_{B}>4 \rho_{0}$ respect to the standard case. Otherwise, in case of superextensive statistical effects $(q>1)$, we can observe a general reduction in the neutrons and the electrons fractions and a small increase of the neutrinos and the hyperons concentrations, with important effects on the softening of the EOS.

In the Fig. 6. particle concentrations for the maximum heating phase $\left(s=2, Y_{\nu_{e}}=0\right)$ are reported. In this condition, due to the higher temperature achieved in the stellar matter, nonextensive statistical effects become more relevant and, consequently, particle concentrations change significantly. When $q<1$, we observe a reduction in the protons and the electrons concentrations and an increase of the neutrons fraction. Whereas, in the super-extensive case $(q>1)$, we have a lower neutrons fraction and an increase of the protons and electrons concentrations. When hyperons are included, Fig. 6, lower panel, we have two main consequences. Firstly, with the absence of neutrinos, the hyperons on-set is shifted at low baryon densities, below $2 \rho_{0}$. Secondly, as a consequence, the electrons and the protons concentrations decrease sensibly with respect to the initial lepton-rich regime $\left(s=1, Y_{L}=0.4\right)$. Therefore, the absence of neutrinos in the stellar matter implies a strong softening of the EOS. In presence of sub-extensive statistics $(q<1)$, hyperons start later with respect to the standard case $(q=1)$, and have in general a bigger concentration at high baryon density. The other way round takes place for super-extensive statistics $(q>1)$.

To better understand the role of nonextensive statistical effects on hyperons formation in the PNS core, in Fig. 7. we report the total hyperons concentration (strangeness per baryon) as a function of the stellar baryon mass (in units of $M_{\odot}$ ) in the lepton rich (upper panel) and in the maximum heating (lower panel) epoch. Although, for $q>1$, hyperons are present at lower baryon densities with
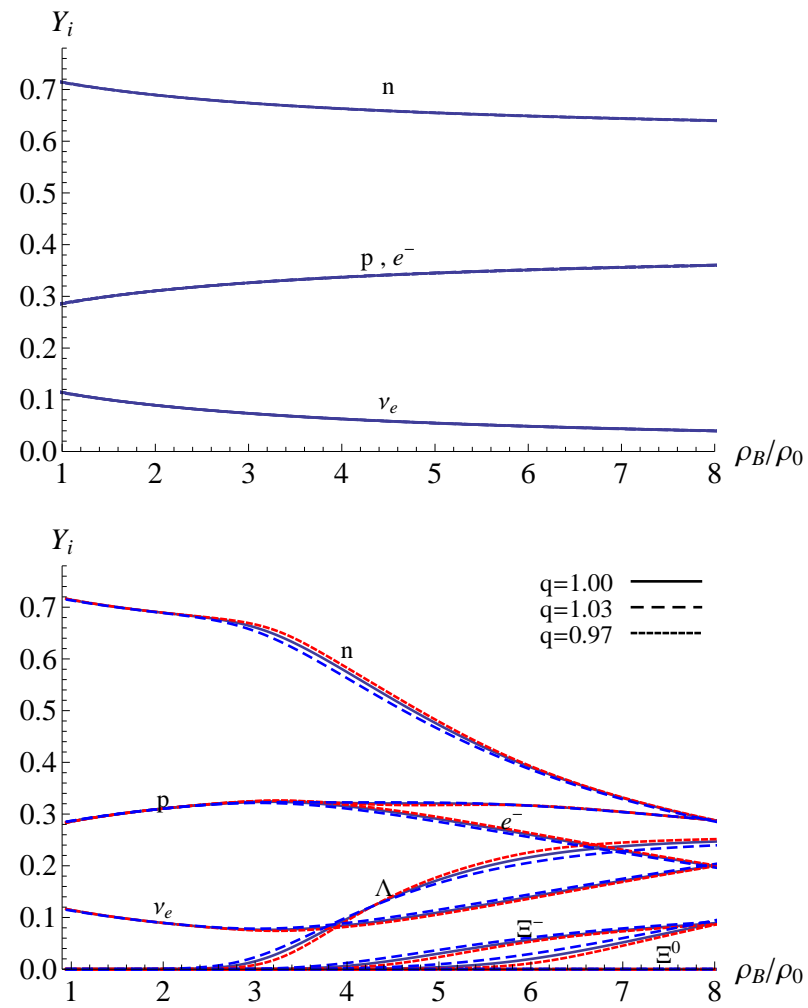

Fig. 5. Particle concentrations $Y_{i}$ without (upper panel) and with hyperons (lower panel) as a function of the baryon density for $s=1$ and $Y_{L}=0.4$.

respect to the standard case $(q=1)$ and a greater hyperons concentration at lower baryon masses is present, we have a significant reduction at higher baryon masses, especially in case of the maximum heating condition. For $q<1$, we have instead the opposite effect: a reduction of the hyperons fraction at low baryon masses and an enhancement at high baryon masses. These features, principally due to the behavior of $\Lambda$ particles concentration as a function of the baryon density (see Fig.s 5 and 6), could imply relevant phenomenological consequences on the evolution of the PNS. In fact, it is known that hyperons significantly increase the neutrino scattering and absorption cross sections 4. Furthermore, the central densities of hyperons stars become progressively larger than that of purely nucleon stars and the evolution timescale of hyperons stars results to be slightly larger because of the smaller mean free path of hyperonic matter. Larger central densities and higher electron neutrino energies, reached in hyperonic PNS, increase the neutrino opacity, temporarily reducing the loss of neutrinos from the stellar core and allow to sustain a higher luminosity at late times.

Finally, in the Table 1, we report the maximum gravitational (baryonic) masses (in units of $M_{\odot}$ ) and the corresponding values of radius and central baryon density, for different PNS configurations. We have taken in consideration different values of the nonextensive $q$-parameter, 

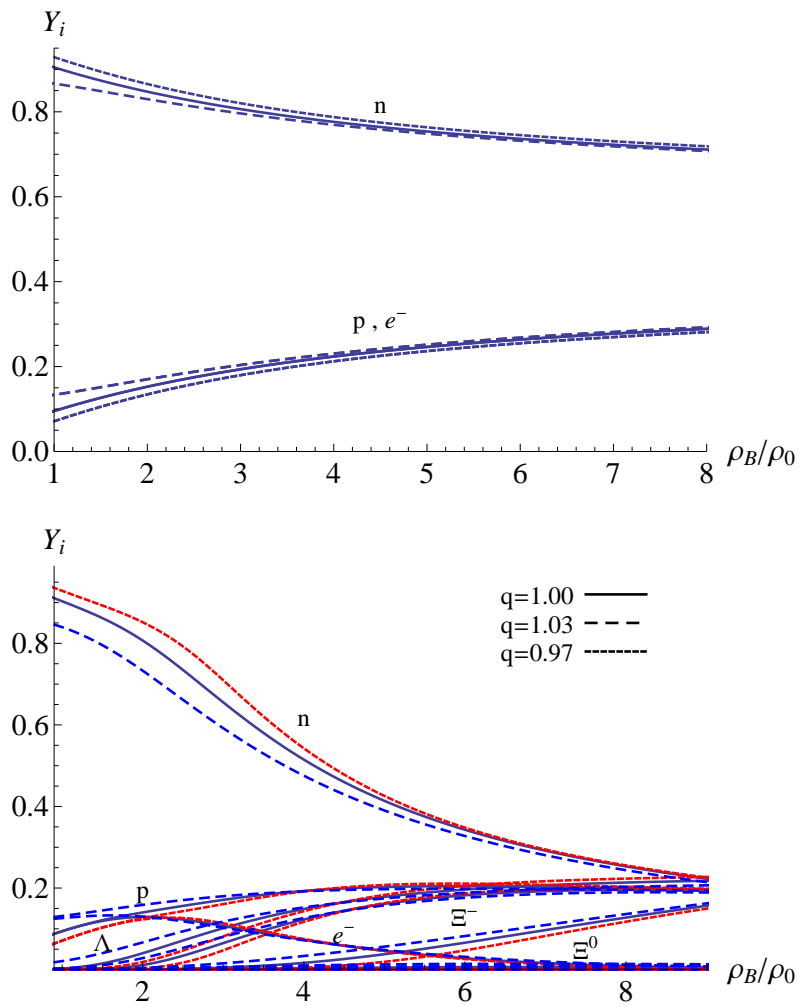

Fig. 6. The same of Fig. 5 for the maximum heating phase $\left(s=2\right.$ and $\left.Y_{\nu_{e}}=0\right)$.

entropy per baryon and leptons concentration, in absence $(n p)$ and in presence $(n p H)$ of hyperons.

As we have already remarked, nonextensive statistical effects result to be most relevant when hyperons are present and the maximum heating phase is achieved $(s=$ $\left.2, Y_{\nu_{e}}=0\right)$. In fact, when the entropy per baryon is equal to one and the matter is lepton rich, the difference in the maximum mass is very small both with and without hyperons. Moreover, immediately after the deleptonization, nonextensive statistical effects become more important, especially in presence of hyperons. The maximum mass is in general decreased when we consider a sub-extensive statistics $(q<1)$, and increased when $q>1$.

Furthermore, in the presence of hyperons, when the stellar core contains non-leptonic negative charges, the maximum masses of neutrino-trapped stars result to be significantly larger than for low temperatures and for lepton poor matter. Hence, there exists a window of initial masses for which the star becomes unstable to gravitational collapse during deleptonization and a black hole can take place [2, 4, 5]. We can see that the formation of such a metastable phase strongly depends on the presence of nonextensive statistical effects and the window of metastability grows with the value of the nonextensive index $q$. In particular, for $q>1$, we still have a very large value of the maximum baryonic mass in the maximum heating phase $\left(s=2, Y_{\nu_{e}}=0\right)$, significantly larger than the one in the
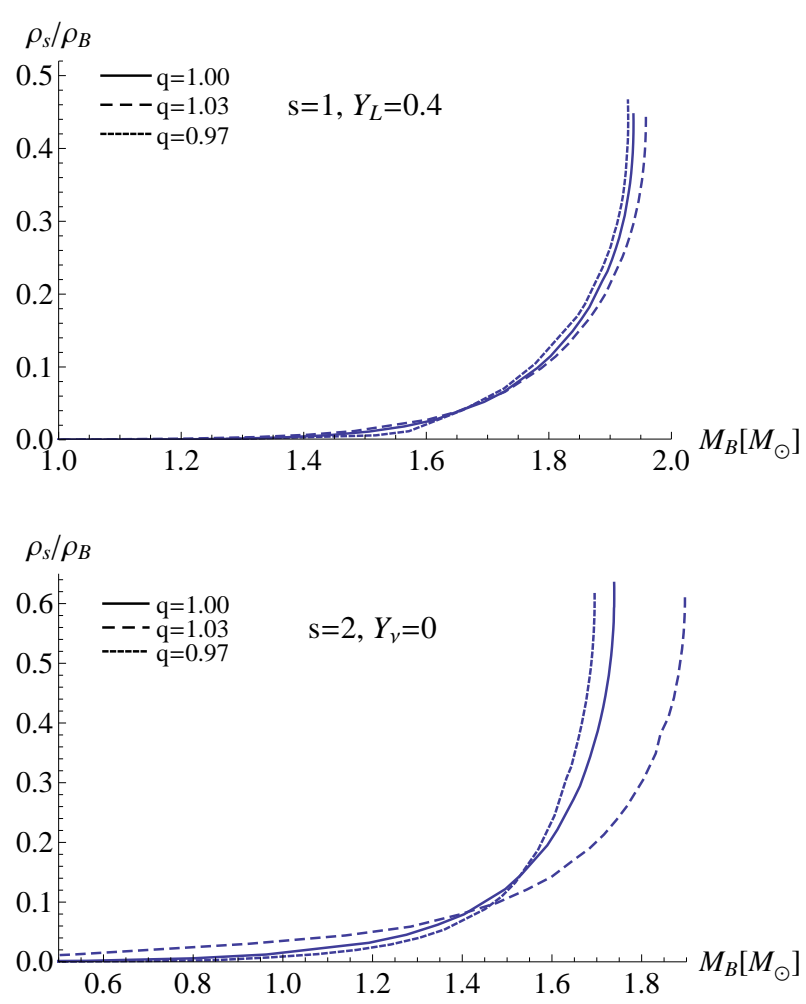

Fig. 7. Hyperons concentration $\rho_{s} / \rho_{B}$ (strangeness per baryon) as a function of the baryon mass $M_{B}$ in the lepton rich (upper panel) and in the maximum heating phase (lower panel).

cold catalyzed condition (where nonextensive statistical effects do not play any role). Therefore, for nucleons-only stars, a black hole could form only after the core bounce, because the maximum mass supported by neutrino-free stars is bigger than that supported for neutrino-rich case. Whereas, in presence of hyperons, a black hole can take place also after the deleptonization era because of the realization of a metastable phase, which becomes more relevant in presence of super-extensive statistical effects.

\section{Conclusions}

We have investigated the physical properties of the PNS in the framework of a relativistic mean field theory based on nonextensive statistical mechanics, characterized by power-law quantum distributions. We have studied the finite temperature EOS in $\beta$-stable matter in absence and in presence of hyperons and trapped neutrinos. From a phenomenological point of view, we have considered the nonextensive index $q$ as a free parameter, even if, in principle, it should depend on the physical conditions inside the PNS, on the fluctuation of the temperature and be related to microscopic quantities (such as, for example, the mean interparticle interaction length). In this context, let us remember that, in the diffusional approximation, a 
Table 1. Maximum gravitational (baryonic) masses $M_{\max }$ (in units of $M_{\odot}$ ) and corresponding values of radius $R$ and central baryon density $\rho_{c}$ in absence $(n p)$ and in presence $(n p H)$ of hyperons for different values of the nonextensive parameter $q$. The results are reported for different values of entropy per baryon and leptons concentration. For completeness, the values for the cold-catalyzed phase $\left(s=0, Y_{\nu_{e}}=0\right)$ for $q=1$ are also reported (nonextensive statistical mechanics does not play any role in this last regime).

\begin{tabular}{|c|c|c|}
\hline & \multicolumn{2}{|c|}{$n p$} \\
\hline$q$ & $s=1, Y_{L}=0.4$ & $s=2, Y_{\nu_{e}}=0$ \\
\hline 0.97 & $\begin{array}{c}M_{\max }=1.96(2.20) \\
\mathrm{R}=10.52 \mathrm{~km} \\
\rho_{c}=7.33 \rho_{0}\end{array}$ & $\begin{array}{c}M_{\max }=2.06(2.34) \\
\mathrm{R}=11.37 \mathrm{~km} \\
\rho_{c}=6.50 \rho_{0}\end{array}$ \\
\hline 1.00 & $\begin{array}{c}M_{\max }=1.97(2.21) \\
\mathrm{R}=10.69 \mathrm{~km} \\
\rho_{c}=7.23 \rho_{0}\end{array}$ & $\begin{array}{c}M_{\max }=2.09(2.38) \\
\mathrm{R}=11.80 \mathrm{~km} \\
\rho_{c}=6.17 \rho_{0}\end{array}$ \\
\hline 1.03 & $\begin{array}{c}M_{\max }=1.98(2.23) \\
\mathrm{R}=10.81 \mathrm{~km} \\
\rho_{c}=7.15 \rho_{0}\end{array}$ & $\begin{array}{c}M_{\max }=2.15(2.47) \\
\mathrm{R}=12.39 \mathrm{~km} \\
\rho_{c}=5.75 \rho_{0}\end{array}$ \\
\hline & \multicolumn{2}{|c|}{$\begin{aligned} s=0, Y_{\nu_{e}}=0 \\
\end{aligned}$} \\
\hline 1.00 & \multicolumn{2}{|c|}{$\begin{array}{c}M_{\max }=2.05(2.39) \\
\mathrm{R}=11.11 \mathrm{~km} \\
\rho_{c}=6.91 \rho_{0} \\
\end{array}$} \\
\hline & \multicolumn{2}{|c|}{$n p H$} \\
\hline $\bar{q}$ & $s=1, Y_{L}=0.4$ & $s=2, Y_{\nu_{e}}=0$ \\
\hline 0.97 & $\begin{array}{c}M_{\max }=1.75(1.93) \\
\mathrm{R}=11.11 \mathrm{~km} \\
\rho_{c}=6.84 \rho_{0}\end{array}$ & $\begin{array}{c}M_{\max }=1.55(1.69) \\
\mathrm{R}=12.39 \mathrm{~km} \\
\rho_{c}=5.59 \rho_{0}\end{array}$ \\
\hline 1.00 & $\begin{array}{c}M_{\max }=1.76(1.94) \\
\mathrm{R}=11.41 \mathrm{~km} \\
\rho_{c}=6.62 \rho_{0}\end{array}$ & $\begin{array}{c}M_{\max }=1.59(1.74) \\
\mathrm{R}=12.75 \mathrm{~km} \\
\rho_{c}=5.44 \rho_{0}\end{array}$ \\
\hline 1.03 & $\begin{array}{c}M_{\max }=1.78(1.96) \\
\mathrm{R}=11.56 \mathrm{~km} \\
\rho_{c}=6.51 \rho_{0}\end{array}$ & $\begin{array}{c}M_{\max }=1.71(1.90) \\
\mathrm{R}=13.34 \mathrm{~km} \\
\rho_{c}=5.05 \rho_{0}\end{array}$ \\
\hline & \multicolumn{2}{|c|}{$s=0, Y_{\nu_{e}}=0$} \\
\hline 1.00 & \multicolumn{2}{|c|}{$\begin{array}{c}M_{\max }=1.57(1.76) \\
\mathrm{R}=12.35 \mathrm{~km} \\
\rho_{c}=5.66 \rho_{0} \\
\end{array}$} \\
\hline
\end{tabular}

value $q \neq 1$ implies the presence of an anomalous diffusion among the constituent particles (the mean square displacement obeys to a power-law behavior $<x^{2}>\propto t^{\alpha}$ with $\alpha \neq 1$ ).

We have shown that nonextensive statistical effects could play a crucial role in the structure and in the evolution of the PNS also for small deviations from the standard Boltzmann-Gibbs statistics. As expected, nonextensive statistical effects result to be particularly important during the maximum heating phase $\left(s=2, Y_{\nu_{e}}=0\right)$, while become less relevant during the initial lepton rich state $\left(s=1, Y_{L}=0.4\right)$ and negligible in the cold cat- alyzed phase $\left(s=0, Y_{\nu_{e}}=0\right)$, due to the low temperatures achieved.

We have studied the relevance of nonextensive statistical effects: i) in the temperature behavior as a function of the baryon density, ii) in the softening of the EOS and, consequently, in the central baryon densities reached in the PNS core at fixed baryon mass, iii) in the lepton chemical potentials, iv) in the particle concentrations, in the hyperons formation and in the strangeness per baryon at fixed total baryon mass, v) in the maximum gravitational and baryonic masses. Such a variation of physical quantities, respect to the standard case, can imply important consequences on the determination of the matter specific heat, on the neutrino mean free path inside the stellar core and, consequently, in the neutrino opacity and luminosity.

We have considered both cases of sub-extensive $(q<1)$ and super-extensive $(q>1)$ statistical effects which entail a sensible difference on the power-law particle distribution in the high energy region. When the entropic $q$ parameter is smaller than one, the energy tail of the particle distribution is depleted, otherwise, when $q$ is greater than one, the energy tail is enhanced. In the PNS context, the physical meaning of the difference between a sub-extensive or a super-extensive statistical behavior is reflected in different and well distinguishable phenomenological PNS properties. For $q<1$, we have a reduction in temperature at fixed baryon density with respect to the standard case $(q=1)$. Especially in the maximum heating phase, the EOS becomes slightly softer and higher central baryon densities at fixed total baryon mass are reached, influencing the neutrino diffusion during the deleptonization process. In the case of sub-extensive effects, hyperons start later but have a bigger concentration at high baryon density, allowing to sustain a higher neutrino luminosity at late times. The other way round takes place in the case of $q>1$. We have an increase of the temperature as a function of the baryon density and lower central densities at fixed baryon mass are reached. The hyperons on-set is shifted at lower baryon densities and a greater hyperons concentration at low baryon masses is present. On the other hand, a significant reduction of the hyperons concentration at high stellar masses take place, contributing to a lower luminosity at late times. Moreover, we have shown that, in presence of super-extensive statistical effects and hyperon degrees of freedom, it is favored the realization of a metastable phase, with an enhancement of a possible black hole formation after the deleptonization era.

\section{References}

1. M. Prakash, J.M. Lattimer, J.A. Pons, A.W. Steiner, S. Reddy, Lect. Notes Phys. 578, 364 (2001).

2. M. Prakash, J.R. Cooke, J.M. Lattimer, Phys. Rev. D 52 , 661 (1995).

3. J.A. Pons, A.W. Steiner, M. Prakash, J.M. Lattimer, Phys. Rev. Lett. 86, 5223 (2001).

4. J.A. Pons, S. Reddy, M. Prakash, J.M. Lattimer, J.A. Miralles, Astrophys. J. 513, 780 (1999).

5. M. Prakash, I. Bombaci, M. Prakash, P.J. Ellis, J.M. Lattimer, R. Knorren, Phys. Rep. 280, 1 (1997). 
6. A. Drago, A. Lavagno, Phys. Lett. B 511, 229 (2001); Z. Berezhiani et al., Astrophys. J. 586, 1250 (2003); A. Drago, A. Lavagno, G. Pagliara, Phys. Rev. D 69, 057505 (2004); A. Drago, A. Lavagno, I. Parenti, Astrophys. J. 659, 1519 (2007).

7. S. Schmidt et al., Phys. Rev. D 59, 094005 (1999).

8. T.S. Biró, C. Greiner, Phys. Rev. Lett. 79, 3138 (1997); T.S. Biró, J. Phys. G: Nucl. Part. Phys. 35, 044056 (2008).

9. C. Tsallis, J. Stat. Phys. 52, 479 (1988); C. Tsallis, R.S. Mendes, A.R. Plastino, Physica A 261, 534 (1998). See also http://tsallis.cat.cbpf.br/biblio.htm for a regularly updated bibliography on the subject.

10. M. Gell-Mann, C. Tsallis, Nonextensive Entropy: Interdisciplinary Applications (New York, Oxford University Press, 2004).

11. C. Tsallis Introduction to Nonextensive Statistical Mechanics (New York, Springer-Verlag, 2009).

12. T. Kodama, H.-T. Elze, C.E. Aiguiar, T. Koide, Europhys. Lett. 70, 439 (2005); H.-T. Elze, T. Kodama, Phys. Lett. A 335, 363 (2005); T. Kodama, T. Koide, Eur. Phys. J. A 40, 289 (2009)

13. I. Bediaga, E.M.F. Curado, J.M. de Miranda, Physica A 286, 156 (2000).

14. W.M. Alberico, A. Lavagno, P. Quarati, Eur. Phys. J. C 12, 499 (2000); A. Lavagno, A.M. Scarfone, P.N. Swamy, J. Phys. A 40, 8635 (2007); A. Lavagno, A.M. Scarfone, P.N. Swamy, Eur. Phys. J. C 47, 253 (2006); A. Lavagno, J. Phys. A 41, 244014 (2008).

15. C. Beck, Physica A 286, 164 (2000); C. Beck, Eur. Phys. J. A 40, 267 (2009).

16. G. Wilk, Z. Włodarczyk, Phys. Rev. Lett. 84, 2770 (2000); G. Wilk, Z. Włodarczyk, Eur. Phys. J. A 40, 299 (2009).

17. A. Lavagno, P. Quarati, Phys. Lett. B 498, 47 (2001); A. Lavagno, P. Quarati, Nucl. Phys B [PS] 87, 209 (2000); F Ferro, A. Lavagno, P. Quarati, Eur. Phys. A 21, 529 (2004); A. Lavagno et al., Astro. Lett. Commun. 35, 449 (1998).

18. T.S. Biró, G. Purcsel, Phys. Rev. Lett. 95, 162302 (2005); T.S. Biró, G. Purcsel, Phys. Lett. A 372, 1174 (2008); T.S Biró, G. Purcsel, K. Ürmössy, Eur. Phys. J. A 40, 325 (2009).

19. A. Lavagno, Physica A 305, 238 (2002); A. Lavagno, Phys. Lett. A 301, 13 (2002); W.M. Alberico, P. Czerski, A. Lavagno, M. Nardi, V Somá, Physica A 387, 467 (2008).

20. J. Cleymans, G. Hamar, P. Levai, S. Wheaton, J. Phys. G: Nucl. Part. Phys. 36, 064018 (2009); J. Cleymans, J. Phys. G: Nucl. Part. Phys. 37, 094015 (2010).

21. S.J. Landau, M.E. Mosquera, H. Vucetich, Astrophys. J. 637, 38 (2006).

22. P. Quarati, A.M. Scarfone, Astrophys. J. 666, 1303 (2007).

23. J.C. Carvalho, J.D. do Nascimento, R. Silva, J.R. Medeiros, Astrophys. J. 696, L48 (2009).

24. G. Livadiotis, D. J. McComas, Astrophys. J. 714, 971 (2010).

25. M.P. Leubner, Astrophys. J. 632, L1 (2005).

26. F.I.M. Pereira, R. Silva, J.S. Alcaniz, Phys. Rev. C 76, 015201 (2007); F.I.M. Pereira, R. Silva, J.S. Alcaniz, Phys. Lett. A 373, 4214 (2009).

27. A. Lavagno, D. Pigato, P. Quarati, J. Phys. G: Nucl. Part. Phys. 37, 115102 (2010); W.M. Alberico, A. Lavagno, Eur. Phys. J. A 40, 313 (2009).

28. A. Burrows, T.J. Mazurek, J.M. Lattimer, Astrophys. J. 251, 325 (1981).

29. O.G. Benvenuto, G. Lugones, Royal Astro. Soc. 304, 25 (2008).
30. A.M. Tewelderberhan, A.R. Plastino, H.C. Miller, Phys. Lett. A 343, 71 (2005).

31. J. Rozynek, G. Wilck, J. Phys. G: Nucl. Part. Phys. 36, 125108 (2009).

32. J.D. Walecka, Ann. of Phys. 83, 491 (1994).

33. J. Boguta and A.R. Bodmer, Nucl. Phys. A 292, 413 (1997).

34. N.K. Glendenning and S.A. Moszkowski, Phys. Rev. Lett. 67, 2414 (1991).

35. M. Di Toro et al., Nucl. Phys. A 775, 102 (2006); L. Bonanno, A. Drago, A. Lavagno, Phys. Rev. Lett. 99, 242301 (2007); A. Drago, A. Lavagno, G. Pagliara, Phys. Rev. D 69, 057505 (2004).

36. A. Lavagno, Phys. Rev. C 81, 044909 (2010).

37. A. Steiner, M. Prakash, J.M. Lattimer, Phys. Lett. B 486, 239 (2000).

38. T. Fischer, S.C. Whitehouse, A. Mezzacappa, F.-K. Thielemann, M. Liebendörfer, A\&A 499, 1 (2009). 